\title{
RECENT ADVANCES IN QUEUEING NETWORKS : A SURVEY WITH APPLICATIONS TO AUTOMATED MANUFACTURING
}

\author{
R. Ram and N. Mswanadham, \\ Department of Computer Science and Automation, \\ Indian Institute of Science, \\ Bangalore 560012
}

\begin{abstract}
Queueing models have been the most widely used analytical modelling tool for performance studies on complex manufacturing systems. Conventional analysis has proceeded along the lines of product form queueing models; and their extensions. Traditional applications of these models have been restricted to the computation of mean values of important performance measures; besides, certain simplifying assumptions have to be made for these models to be applicable. The last decade has witnessed important advances in the applicability of these traditional queueing networks, as well as in newer queueing models that exhibit constructs capable of modelling generic issues arising in a real-life manufacturing system. The issues taken up in this paper are: (i) computation of higher moments of performance measures, besides mean values; (ii) the phenomenon of blocking due to limitations of buffer capacities, and (iii) analysis of a network of multiqueues model of an Automated Manufacturing System. We survey the recent advances in these three modelling issues which are of great importance in the manufacturing context, and present analytical models of Automated Manufacturing Systems incorporating these features. Each model is illustrated with a numerical example.
\end{abstract}

\section{Introduction}

Automated Manufacturing Systems (AMSs) are a class of advanced manufacturing systems, employing versatile workcentres and a highly capable Material Handling System (MHS) for economical, high quality production of a wide variety of part types. The highly capital intensive nature of these systems necessitates the use of powerful performance modelling tools during both the design and operational phases.

Queueing models are the primary analytical modelling tool for the performance analysis of AMSs. In particlar, product form queueing models ([1]), a special class of analytically tractable queueing networks, have been the most popular models, apart from several heuristic (approximate) analyses of more general (non- product form) queueing networks. The last decade has seen several significant developments in the efficient and accurate analysis of general queueing network models arisisng in various contexts, especially the performance evaluation of advanced computer systems and computer communication systems. In this paper, we survey these developments from the perspective of performance modelling of AMSs. These new results facilitate elegant modelling of generic features that are typical in Automated Manufacturing. We shall highlight the applicability of important recent results in the following three areas for the performance modelling of AMSs:
- Computation of second and higher moments of performance measures, such as inventory levels (queue lengths), and manufacturing lead times (response times). In certain situations, such as choosing between design alternatives, higher moment information (like variance) of these performance measures is also required, apart from mean values.

- Blocking due to finite queue capacities: conventional queueing models assume that every queue in the model has no capacity constraints. This is unrealistic, since in a real life manufacturing system, there is only a finite number of buffers for holding waiting workpieces in front of a machine. Hence, an upstream machine cannot proceed with processing (gets blocked) if the buffers in the succeeding downstream machine are all occupied.

- Multiqueue models of nodes (machines) and networks of multiqueues: Traditional modelling has proceeded assuming a single queue at each node (machining centre); owing to the presence of multiple part types, multiqueue models of nodes, with each part type forming a separate queue are more realistic models. The manufacturing system may be represented as a network of such multiqueue nodes.

The rest of this paper is organised as follows: in section 2, we briefly discuss the advances in computational algorithms for product form networks, with particular attention to the calculation of higher moments of performance measures. Section 3 deals with the analysis of queueing models exhibiting blocking. In section 4, we present an analysis of a simple 'network of multiqueues' model of an AMS. We conclude with a brief look at possible refinements of and extensions to the models presented in this paper.

\section{Product-form models: Computation of higher moments of performance measures}

In a product-form queuing network (PFQN) model of a manufacturing system, the each machine is represented by $a$ node and each part type is represented by separate customer class. PFQNs can be solved for the important performance measures by- the well known convolution ([2]) and mean value analysis algorithms ([3]). The computational requirements of these alogrithms are exponential in the number of part types (chains); hence the application of these algorithms are infeasible for systems with many part types or many workpieces (large populations). 
Newer computational algorithms like Recursion by Chain Algorithm (RECAL, [4]), Mean-Value Analysis by Chain (MVAC, [5]) and Direct Analysis by Chain (DAC, [6]) have computational requirements that are polynomial in the number of part types, for a given number of machine centres; these are more suitable for analysing manufacturing systems than the conventional algorithms since there are usually more part types than machines. A comprehensive survey of queueing model applications to performance modelling appears in [7]. Several approximate algorithms have also been developed for quick analysis of PFQNs.

An important issue in the analysis of PFQNs is the computation of the higher moments of performance measures apart from the mean value. However, due to the very high computational requirements of the exact methods, it is necessary to take recourse to approximations. McKenna and Mitra ([8]) have developed an algorithm based on asymptotic expansion of the partition function of the network; Strelen $([9,10])$ has developed an approximate analysis (Moment Analysis) based on the Linearizer algorithm $([11]$ of Chandy and Neuse.

\subsection{Moment Analysis: A manufacturing ap- plication}

Let us consider an AMS consisting of $\mathrm{K}$ workcentres (numbered 1 to $\mathrm{K}$ ), and a MHS (called station 0 ), represented by the wellknown closed, central server queueing model. The central server represents the MHS, and each of the workcentres is represented by a sepatate queue (Fig. 1). From the MHS, a workpiece is routed to machine centre $\mathrm{k}$ with probability $p_{k}$, or it leaves the system with probability $p_{0}$ and is immediately replaced by an identical workpiece; $\left(\sum_{k=0}^{K} p_{k}=1\right)$. After being processed at the workcentre, the workpiece is always routed back to the MHS. For simplicity, assume a single part type of which $\mathrm{N}$ identical workpieces are present in the system. All stations work in a fixedrate-FCFS fashion, with an exponentially distributed machining time (or move time at the MHS) of mean $x_{k}$ at station $\mathrm{k}$. The mean values of the performance measures may be computed by the well known PFQN algorithms; let us concentrate on obtaining the second moment of the mean queue lengths. (Although higher moment information may be computed using some exact computational algorithms also, in general the computational requirements make exact analysis infeasible, especially when there are multiple chains). We use the exact Moment Analysis method of Strelen ([10]); for systems with a large number of workpieces or a number of part types, it may be necessary to use approximations like Strelen's Moment Analysis-Linearizer ([10]).

Let $e_{k}$ denote the visit ratio of a workpiece at station $\mathbf{k}$; for our example, $e_{k}=1 / p_{0}$ for $k=0$ and $e_{k}=p_{k} / p_{0}$ for $1 \leq k \leq K$, from well known results of PFQN theory. Let $Q_{k}^{(j)}(n)$ and $W_{k}^{(j)}(n)$ respectively denote the expected value of the $j^{\text {th }}$-moment of the queue length and the wating time at station $\mathrm{k}(0 \leq k \leq K)$, when there are $n$ workpieces in the system $(0 \leq n \leq N)$. Let $T^{(j)}(n)$ denote the $j^{\text {th }}$-moment of the system throughput with $\mathrm{n}$ workpieces. Let $\rho_{k}=e_{k} \times x_{k}$ denote the relative traffic intensity at station $\mathrm{k}$. Let us restrict our attention to the second moment of the mean queue lengths only. These may be computed by the (exact) Moment Analysis algorithm ([9]) as follows.

(1) Initialisation: $Q_{k}^{(j)}(0)=W_{k}^{(j)}(0)=T^{(j)}(n)=0, j=$ $1,2,0 \leq k \leq K$

$\frac{\partial Q_{l}^{(1)}(0)}{\partial x_{l}}=\frac{\partial W_{k}^{(1)}(0)}{\partial x_{l}}=\frac{\partial T^{(1)}(0)}{\partial x_{l}}=0$, for $0 \leq k, l \leq K$.

(2) Computations: for $n=1$ to $N$ do begin

$$
\begin{aligned}
& \text { 2.1. } W_{k}^{(1)}(n)=\rho_{k}\left(1+Q_{k}^{(1)}(n-1)\right), 0 \leq k \leq K \\
& \text { 2.2. } T^{(1)}(n)=n /\left[\sum_{l=0}^{K} W_{l}^{(1)}(n)\right], 0 \leq k \leq K \\
& \text { 2.3. } Q_{k}^{(1)}(n)=T^{(1)}(n) W_{k}^{(1)}(n), 0 \leq k \leq K
\end{aligned}
$$

2.4. for $0 \leq k, l \leq K d o$

begin

$$
\begin{aligned}
\frac{\partial W_{k}^{(1)}(n)}{\partial x_{l}}= & e_{k}\left[\delta_{k, l}\left\{1+Q_{k}^{(1)}(n-1)\right\}+\right. \\
& \left.x_{k} \frac{\partial W_{k}^{(1)}(n-1)}{\partial x_{l}}\right] \\
\frac{\partial T^{(1)}(n)}{\partial x_{k}}= & (-n) /\left[\sum_{j=0}^{K} W_{j}^{(1)}(n)\right]^{2} \times \sum_{j=0}^{K} \frac{\partial W_{j}^{(1)}(n)}{\partial x_{l}} \\
\frac{\partial Q_{k}^{(1)}(n)}{\partial x_{l}}= & W_{k}^{(1)}(n) \frac{\partial T^{(1)}(n)}{\partial x_{l}}+\frac{\partial W_{k}^{(1)}(n)}{\partial x_{l}} T^{(1)}(n) \\
Q_{k}^{(2)}(n)= & x_{k} \frac{\partial Q_{k}^{(1)}(n)}{\partial x_{k}}+\left[Q_{k}^{(1)}(n)\right]^{2}
\end{aligned}
$$

end; $\{$ of step 2.4\}

end. $\{$ of step 2.\}

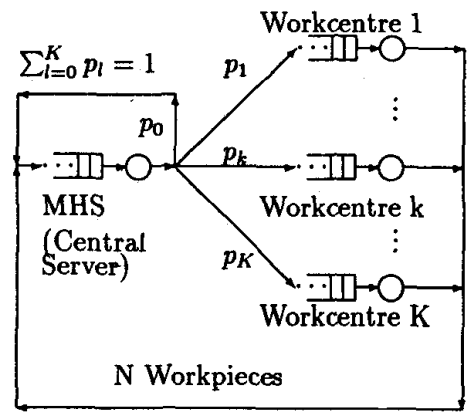

Figure 1: The Central Server Model of an AMS 


\begin{tabular}{|c|c|c|c|c|}
\hline $\begin{array}{c}\text { Station, } \\
\text { k }\end{array}$ & Through- & Waiting & \multicolumn{2}{|c|}{ Queue Length } \\
put, $T_{k}^{(1)}$ & time, $W_{k}^{(1)}$ & $Q_{k}^{(1)}$ & $Q_{k}^{(2)}$ \\
\hline 0 & 0.41316 & 1.6881 & 0.6795 & 1.6387 \\
\hline 1 & 0.12395 & 17.5688 & 7.2587 & 57.7477 \\
\hline 2 & 0.10329 & 3.7581 & 1.5527 & 5.8344 \\
\hline 3 & 0.16526 & 1.8889 & 0.4912 & 0.9640 \\
\hline
\end{tabular}

Table 1: Results of Moment Analysis for exampe AMS

\subsection{Numerical example}

Consider a manufacturing system with $K=3$ workcentres apart from the MHS, with $\mathrm{N}=10$ worpieces. Let the routing probabilities be: $p_{0}=0.05 ; p_{1}=0.3 ; p_{2}=0.25 ; p_{3}=0.4$; the mean processing times are $x_{0}=1, x_{1}=8, x_{2}=6$, and $x_{3}=2$. Table 1 gives the mean values of the throughput, the waiting time, and queue length along with the second moment of the queue length.

\section{Queueing models with blocking}

An important feature of manufacturing systems in general, and AMSs in particular, is the availability of only a limited number of buffers space to hold waiting workpieces in front of a workcentre, due to either a physical limitation of storage space, or the provision of a limited number of fixtures, or a combination of both. Hence, workcentres have to be represented by a queue with finite capacity; this gives rise to the phenomena of blocking: e.g., when a workpiece completes processing at a particular workcentre (say k), all the buffers at the next workcentre (say l) in its routing may be full; hence, workcentre $\mathrm{k}$ cannot proceed with the processing of the next workpiece until this workpiece is moved out, which is possible only when a free buffer is available at workcentre 1 . This is one particular type of blocking called type 1 blocking or transfer blocking or manufacturing blocking. Traditional queueing models (including PFQNs) of AMSs assume infinite capacity queues at all nodes; queueing networks with finite capacity queues model AMSs to a greater degree of detail. However, exact analysis of queueing networks with finite buffers (i.e., queueing networks with blocking) can be carried out only for some special cases. More general systems can be analysed only approximately. Also, most of the analyses in the literature deal with networks featuring a single part type (job class) only. The two very recent survey articles by Perros ([12]) and Onvural ([13]) comprehensively scan the literature on finite capacity open and closed queueing networks respectively. The central server model, with the central server (MHS in our example) having a infinite capacity queue, the stations having finite or infinite capacity queues, with exponential server assumption, with a single part type, under manufacturing blocking has been shown to have a product-form solution ([14]). This representation is reasonable since usually machines have very limited buffer capacity, and the MHS has the capacity to hold all the workpieces on the shop floor. The analysis of this system has been extended to the multiple job classes version by Zhuang and Hindi ([15]). Computational algorithms for finite capacity queueing networks with a different blocking mechanism called rejection blocking are presented by Akyildiz and von Brand ([16]).

An example

Continuing with the central server model of the example system presented in the previous section, let us assume finite capacity buffers at each of the K workcentres; the MHS (central server) has an infinite capacity queue. We analyse the system

\begin{tabular}{|c|c|c|c|c|}
\hline Station, $\mathbf{k}$ & Queue Len. & Util. & T'put & Wait. Time \\
\hline 0 & 5.949 & 0.419 & 0.12581 & 14.185 \\
\hline 1 & 2.721 & 0.999 & 0.10484 & 17.265 \\
\hline 2 & 1.398 & 0.629 & 0.16775 & 13.333 \\
\hline 3 & 0.481 & 0.335 & 0.41939 & 2.868 \\
\hline
\end{tabular}

Table 2: Results for example AMS featuring blocking

by the approximate mean value analysis of blocking queuing networks (MVABLO) developed in [17]. Let the buffer capacity of workstations 1,2 and 3 be 4 units each (including the server) and that of the MHS be 7. The routing probabilities and the processing times are the same as in the previous example. Table 2 gives the mean values of the throughput, queue length, utilisation and waiting time at each of the stations

\section{Network of multiqueue nodes}

\subsection{Polling models of workcentres and the MHS}

The fundamental machining unit in an AMS is a versatile workcentre, capable of processing different types of parts. In traditional queueing models, we assume that different parts types wait in a common queue. Besides, we assume a common processing time for the different part types, i.e., treat them different part types as homogenous, otherwise the analysis becomes very difficult. Due to the inherent differences among part types, it is more realistic to treat them as distinct, by assuming (logically) a separate queue for each part type. Hence, we may model a node (machining centre) in the AMS by a single server attending to multiple queues. If there are $\mathrm{N}$ part types being processed at a workcentre, usually they are taken up for machining in the cyclic order $1,2, \ldots, N-1, N, 1,2$, etc. When the machine changes over from processing part type $\mathrm{j}$ to processing part type $(j+1)$ modulo $N+1$, a setup operation is carried out for part type $\mathrm{j}+1$, before starting machining the workpieces. Such cyclic server models of the workcentres are a special class of the more general polling models; see [18] for a survey on the analysis of polling models. Since the MHS moves the workpieces among the machine centres, it may also be considered (in a global sense) as a polling model, with one queue corresponding to each machining centre that inputs workpieces for handling. Hence, an AMS can be modelled as a network of such multiqueue models, interconnected by the MHS. This is analogous to the interconnection of token passing Local Area Networks by a high-speed backbone network: the LANs are analogous to the machining centres in the AMS, and the backbone network corresponds to the MHS.

The exact analysis of such systems (interconnected networks of polling models) has not been done as yet in the literature. However, approximate analyses, utilising simplifying assumptions have been presented, e.g., Kuruppillai and Bengston ([19]), and Ibe and Cheng $([20])$. The difficulty in the analysis is due to the fact that the arrival process to the MHS (analogously, the backbone network) is not exponential as required for an exact analysis of polling models; to simplify matters, the exponential assumption is usually adapted. Next we present a simple model of an AMS as network of multiqueue nodes, and develop an approximate analysis. 


\subsection{A typical configuration}

Consider an AMS consisting of K versatile workcells, $M_{1}$ through $M_{K}$, one inspection station, and a Automated Guided Vehicle (AGV) system (MHS), and manufacturing $N$ different part types (Fig. .2). Workpieces of part type $\mathrm{j}(1 \leq j \leq N)$ arrive to the system according to Poisson process of parameter $\lambda_{j}$. An arriving workpiece is sent to workcell $\mathrm{k}(1 \leq k \leq K)$ with probability $p_{k, j}$, where $p_{k, j} \geq 0$ and $\sum_{k=1}^{K} p_{k, j}=1$; thus the arrival rate for part type $\mathrm{j}$ at machine $M_{k}(1 \leq k \leq K)$ is $p_{k, j} \lambda_{j}$. The setup time and processing time for part type $\mathrm{j}$ at workcentre $M_{k}$ are denoted as $S_{k, j}$ (with mean $r_{k, j}$ and variance $\delta_{k, j}$ ) and $B_{k, j}$ (with mean $b_{k, j}$ and second moment $b_{k, j}^{(2)}$ ) respectively. There is a separate input queue for each part type at each workcell; all $\mathrm{K}$ workcells operate in cyclic server fashion described above and process atmost one waiting workpiece of each part type in each cycle (cyclic server with 1-limited service). After processing at the workcells, the workpieces wait in a output queue common to all part types. The AGV system moves the finished workpieces from the workcells to the corresponding queue of each part type at the inspection station; the MHS also works in a cyclic server fashion, moving workpieces out from the buffers of the workcells in the cyclic order $M_{1}, M_{2}, \ldots, M_{K}, M_{1}$ etc.; the MHS works on each output buffer in a exhaustive fashion (i.e., it empties the output buffer of one workcell before taking up the next one). Let $B_{k}^{A G V}$ denote the time for the AGV system to move a workpiece (irrespective of part type) from workcell $M_{k}$ to the inspection station, and $S_{k}^{M H S}$ the time for the AGV system to move over to cell $M_{k}$ after finishing with cell $M_{k-1}$. The inspection station operates in the same fashion as the workcells, examining one workpiece of each part type per cycle. Let $B_{j}^{I N S}$ denote the inspection time for a type $\mathrm{j}$ workpiece, and $S_{j}^{I N S}$ be the time taken by the inspection station to changeover from part $j-1$ to part $\mathrm{j}$.

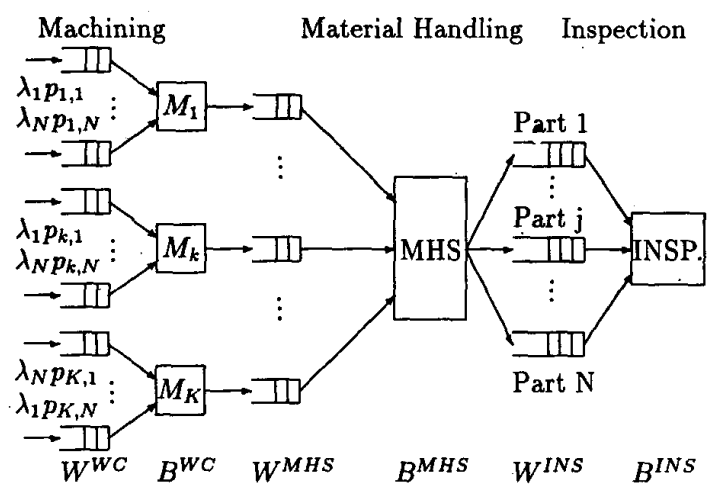

Figure 2: AMS modelled as a Network of Multiqueues

\subsection{Approximate analysis for manufacturing lead time}

We are interested in determining the mean manufacturing lead time (called $T_{j}^{M L T}$ ), for each part type $\mathrm{j}$; this value is defined as the time from the instant the workpiece enters the system to the instant it leaves the system. In our model, the mean manufacturing lead time of an arbitrary part type may be obtained from:

$$
\begin{aligned}
E\left[T^{M L T}\right]= & E\left[W^{W C}\right]+E\left[B^{W C}\right]+E\left[W^{M H S}\right]+E\left[B^{M H S}\right] \\
& +E\left[W^{I N S}\right]+E\left[B^{I N S}\right]
\end{aligned}
$$

where $E\left[W^{W C}\right], E\left[W^{M H S}\right]$ and $E\left[W^{I N S}\right]$ are respectively the mean waiting time at the input queue to the workcell to which it is initially sent, the mean waiting time for the AGV system at the output queue of this workcell, and the mean waiting time in the inspection station queue; $E\left[B^{W C}\right], E\left[B^{M H S}\right]$ and $\left[B^{I N S}\right]$ are respectively the mean time for processing, part movement to the inspection station, and the inspection time at these three stages.

Let $W_{k, j}^{W C}$ denote the waiting time for part type $\mathrm{j}$ at the workcentre $\mathrm{k}, W_{k}^{M H S}$ be the waiting time for an arbitrary part type at the output queue of wokstation $\mathrm{k}$, and $W_{j}^{I N S}$ be the waiting time of a type $j$ workpiece at the inspection station. Since a type $j$ workpiece is probabilistically routed to one of the workcells initially, we obtain the, mean manufacturing lead time for part $j$ as

$$
\begin{aligned}
E\left[T_{j}^{M L T}\right]= & \left\{\sum _ { k = 1 } ^ { \# } p _ { k , j } \left[E\left[W_{k, j}^{W C}\right]+E\left[B_{k, j}\right]+E\left[W_{k}^{M H S}\right]+\right.\right. \\
& \left.\left.E\left[B_{k}^{M H S}\right]\right]\right\}+E\left[W_{j}^{I N S}\right]+E\left[B_{j}^{I N S}\right]
\end{aligned}
$$

In the above equation, we need to compute only the three mean waiting times, which may be done as follows:

- $W_{k, j}^{W C}$ : The mean arrival rate of part type $\mathrm{j}$ to workcell $\mathrm{k}$ is $\lambda_{k, j}=p_{k, j} \lambda_{j}$; using the known values of the setup and processing times, we can compute the mean waiting time by any of the approximate methods; we used the one suggested by Boxma and Meister ([21]).

- $W_{k}^{M H S}$ : We assume that the arrival of workpieces to the output queue of each workcell is exponential, with rate equal to the sum of the arrival rates of diffetrent workpieces to that workcell; i.e., $\lambda_{k}^{M H S}=\sum_{j=1}^{N} \lambda_{k, j}$. Now, the mean waiting time in the output queue of workcell $\mathrm{k}$ can be found by solving the exhaustive service cyclic server model of the MHS by the method presented in [18].

- $W_{j}^{I N S}$ : The arrival of workpieces of each part type to its corresponding queue at the inspection station from the AGV system is assumed to be exponential, with rate equal to the external arrival rate of workpieces to the system, i.e., $\lambda_{j}^{I N S}=\lambda_{j}$. The mean waiting time at the inspection station is obtained by using the same methods as for $W_{k, j}^{W C}$.

\subsection{Numerical example}

Consider an AMS with the configuration consisting of $K=2$ workcells, one AGV, and one inspection station, and producing $N=3$ different types of parts. The following table gives the mean values of the interarrival time, the setup time and processing (inspection) time a workpiece of each part type on the workcells and the inspection station. For simplicity, we assume that all activities take an exponential duration. 


\begin{tabular}{|c|c|c|c|c|c|c|}
\hline \multirow{2}{*}{ art } & \multicolumn{3}{|c|}{ Setup Time } & \multicolumn{3}{c|}{ Processing Time } \\
\cline { 2 - 7 } & M1 & M2 & INSP. & M1 & M2 & INSP \\
\hline 1 & 4.0 & 5.0 & 1.0 & 2.0 & 2.0 & 1.0 \\
\hline 2 & 7.0 & 5.0 & 1.0 & 3.0 & 4.0 & 2.0 \\
\hline 3 & 3.0 & 8.0 & 1.0 & 1.0 & 2.0 & 2.0 \\
\hline
\end{tabular}

The following table gives the routing probabilities, the mean interarrival times, and the time taken by the AGV to move a workpiece from the workcells to the inspection centre. The time taken by the AGV to changeover from one workcell to the next is assumed to be 50 units.

\begin{tabular}{|c|c|c|c|c|c|}
\hline \multirow{2}{*}{ Part } & \multicolumn{2}{|c|}{ Routing Prob } & Mean Inter- & \multicolumn{2}{c|}{ AGV Move Time } \\
\cline { 2 - 3 } & To M1 & To M2 & arrival Time & M1 & M2 \\
\hline 1 & 0.5 & 0.5 & 25.0 & 7.0 & 10.0 \\
\hline 2 & 0.6 & 0.4 & 30.0 & 7.0 & 10.0 \\
\hline 3 & 0.7 & 0.3 & 50.0 & 7.0 & 10.0 \\
\hline
\end{tabular}

The mean manufacturing lead times for the three part types obtained from equations (1) and (2) are:

Mean Manufacturing Lead Times

\begin{tabular}{|c|c|c|c|c|}
\hline Part type & $E\left[W^{W C}\right]$ & $E\left[W^{M H S}\right]$ & $E\left[W^{I N S}\right]$ & Mean MLT \\
\hline 1 & 19.827 & 147.098 & 3.038 & 181.46 \\
\hline 2 & 17.725 & 146.586 & 3.046 & 180.96 \\
\hline 3 & 14.383 & 146.074 & 2.808 & 174.43 \\
\hline
\end{tabular}

\section{Conclusion}

In this paper, we highlighted the application of queueing network models for the performance evaluation of AMSs, addressing separately the issues of computation of higher moments of performance measures, blocking phenomena, and analysis of a open network of multiqueues model of AMS. Due to paucity of space, we had to omit most of the mathematical details. Several interesting and important extensions to the models presented here are possible, like the analysis of a network of multiqueues model with finite buffers, computation of higher moments of performance measures for this model, analysis of a closed network of multiqueues model, and incorporation of the issue of equipment failure and repair in the model. Development of analysis methodologies for these systems, which model AMSs to a greater degree of detail, constitute important unsolved problems in the area of performance evaluation, and is part of our continuing work in this area.

\section{References}

[1] F. Baskett, K. M. Chandy, R. R. Muntz, and F. G. Palacios, "Open, closed and mixed networks of queues with different classes of customers," $J l . A C M$, vol. 22 , no. 2, pp. 248-260, 1975.

[2] M. Reiser and H. Kobayashi, "Queueing networks with multiple closed chains: Theory and computational algorithms," IBM Jl. Res. \& Dev., vol. 19, pp. 283-294, 1975.

[3] M. Reiser and S. S. Lavenberg, "Mean-value analysis of closed multichain queueing networks," Jl. ACM, vol. 27, no. 2, pp. 313-322, 1980.
[4] A. E. Conway and N. D. Georganas, "RECAL-A new efficient algorithm for the exact analysis of multiplechain closed queueing networks," Jl. ACM, vol. 33, pp. 768-791, October 1986.

[5] A. E. Conway, E. de Souza e Silva, and S. S. Lavenberg, "Mean value analysis by chain of product form queueing networks," IEEE Trans. Computers, vol. 38, pp. 432-442, 1989.

[6] E. de Souza e Silva and S. S. Lavenberg, "Calculating joint queue-length distributions in product-form queueing networks," Jl. $A C M$, vol. 36, pp. 194-207, January 1989.

[7] S. S. Lavenberg, "A perspective on queueing models of computer performance," Perf. Evaln., vol. 10, pp. 53-76, 1989.

[8] J. McKenna and D. Mitra, "Asymptotic expansions and integral representations of moments of queue lengths in closed markovian networks," Jl. ACM, 1984.

[9] J. C. Strelen, "A generalisation of mean value analysis to higher moments: Moment analysis," ACM SIGMETRICS Perf. Evaln. Review, vol. 14, no. 1, pp. 129-140, 1986.

[10] J. C. Strelen, "Moment analysis for closed queueing networks and its linearizer," Perf. Evaln., vol. 11, pp. 127-142, 1990.

[11] K. M. Chandy and D. Neuse, "Linearizer: A heuristic algorithm for queueing network models of computing systems," Commn. ACM, vol. 25, no. 2, pp. 126-134, 1982.

[12] H. G. Perros, "Approximation algorithms for open queueing networks with blocking," in Stochastic Analysis of Computer and Communication Systems (H. Takagi, ed.), pp. 451-498, Elsevier Science Publishers B.V. (North-Holland), 1990.

[13] R. O Onvural, "Survey of closed queueing networks with blocking," ACM Computing Surveys, vol. 22, pp. 83-121, June 1990.

[14] D. D. Yao and J. A. Buzacott, "Modeling a class of flexible manufacturing systems with reversible routing," Oper. Res., vol. 35, pp. 87-93, January-February 1987.

[15] L. Zhuang and K. S. Hindi, "Mean value analysis for multiclass closed queueing network models of flexible manufacturing systems with limited buffers," Eur. Jl. Oper. Res., 1990 .

[16] I. F. Akyildiz and H. von Brand, "Computational algorithms for networks of queues with rejection blocking," Acta Informatica, vol. 26, pp. 559-576, 1989.

[17] I. F. Akyildiz, "Mean value analysis for blocking queueing networks," IEEE Trans. Software Engineering, vol. 14, pp. 418-428, April 1988.

[18] H. Takagi, "Queuing analysis of polling models," ACM Computing Surveys, vol. 20, no. 1, pp. 5-28, 1988.

[19] R. Kuruppillai and N. Bengston, "Performance analysis in local area networks of interconnected token rings," Computer Communications, vol. 11, pp. 59-64, April 1988.

[20] O. C. Ibe and X. Cheng, "Analysis of interconnected systems of token ring networks," Computer Communications, vol. 13, pp. 136-142, April 1990.

[21] O. J. Boxma and B. W. Meister, "Waiting-time approximations for cyclic-service systems with switchover times," Perf. Evaln., vol. 7, pp. 299-308, 1987. 